\title{
Periodic Limb Movements in Complex Sleep Apnea Syndrome
}

\author{
Snigdha S. Pusalavidyasagar ${ }^{1}$, Tomasz J. Kuzniar ${ }^{2}$, Eric J. Olson ${ }^{3}$ and Timothy I. Morgenthaler*, ${ }^{3}$ \\ ${ }^{1}$ Division of Pulmonary, Allergy, Critical Care and Sleep Medicine, University of Minnesota, Minneapolis, MN, USA; \\ ${ }^{2}$ Division of Pulmonary and Critical Care Medicine, NorthShore University Health System, Evanston, IL, USA; ${ }^{3}$ Center \\ for Sleep Medicine, Division of Pulmonary and Critical Care Medicine, Mayo Clinic, Rochester, MN, USA
}

\begin{abstract}
Introduction: The development of repetitive central apneas during application of CPAP in patients initially showing obstructive apneas, a condition called complex sleep apnea syndrome (CompSAS), implies respiratory control instability. Respiratory control is known to be transiently destabilized by arousals. We hypothesized that PLMS and PLMrelated arousals would be more frequent in patients with CompSAS compared to those with obstructive sleep apnea (OSA) and could account for some of the respiratory instability.
\end{abstract}

Methodology: Comparative retrospective review of patients studied in our Sleep Disorders Center.

Results: 88 patients with CompSAS were compared with 112 patients with OSA. Total arousal index (TAI), respiratoryrelated arousal index (RRAI), and PLM-related arousal index (PLMAI) were similar during the diagnostic polysomnography of CompSAS and OSA patients. Following CPAP application, patients with CompSAS had a higher TAI [27.2 (15.5 - 39.9) vs. 16.6 (10.7 - 26.5); $\mathrm{p} \leq 0.001$; median (interquartile range)], but a lower PLMI [0 (0 - 21.1) vs. 12.6 (0 - 36.2 ); $\mathrm{p}=0.009]$ and PLMAI [0 $(0-1.3)$ vs. $0.8(0-4.2) ; \mathrm{p}=0.004]$ than patients with OSA.

Conclusion: PLMS and PLM-related arousals are not more common in CompSAS than in OSA patients and do not appear to play a significant role in destabilizing respiratory control in CompSAS patients.

Keywords: Obstructive sleep apnea-hypopnea syndrome, complex sleep apnea syndrome, periodic limb movements, periodic limb movement arousal index, continuous positive airway pressure.

\section{INTRODUCTION}

Periodic limb movements during sleep (PLMS) are a frequent finding in polysomnography, and they are present in a high percentage of the general population [1-3]. They are associated not only with sleep disorders such as sleep apnea syndromes [4], restless legs syndrome (RLS) [5], rapid eye movement (REM) sleep behavior disorder [6], and narcolepsy [7], but also may occur in a wide range of neurological and psychiatric conditions. A high prevalence of PLMS (22$52 \%$ ) is found in patients with congestive heart failure, with reported mean periodic limb movement indexes (PLMI) in the range of 55-73/hour [8, 9]. It is not established whether PLMS play a role in aggravation or causation of central sleep apnea. However, PLMS may be associated with EEG microarousals, changes in the EEG spectral activity, heart rate and blood pressure [10,11], and we and others have observed that repetitive arousals related to PLMS may destabilize breathing and give rise to repetitive periodic central hypopneas and apneas [12].

Some patients with sleep disordered breathing express both central sleep apnea behaviors, a disorder generally thought to be due to dysfunction of central respiratory control, and obstructive apnea and hypopneas, often attributed in large part to anatomic factors [13, 14]. A not uncommon manifestation of this situation, termed the complex sleep

*Address correspondence to this author at the Sleep Disorders Center, Mayo Clinic, 200 First St SW, Rochester, MN 55905, USA; Tel: 507-264-3764; Fax: 507-266-7772; E-mail: morgenthaler.timothy@mayo.edu apnea syndrome (CompSAS), is when patients first demonstrating obstructive apneas and hypopneas manifest frequent central apnea events while on continuous positive airway pressure (CPAP). In recent reports, the mean apnea hypopnea index (AHI) prior to CPAP, predominantly composed of obstructive events, ranged from 32.3-72.1, while the residual AHI and central apnea index (CAI) on treatment ranged from 15.8-48.8 and 10.1-26.2, respectively [15-17]. The pathophysiologic mechanisms underlying the development of central apneas are not clear. Since PLMS may cause repetitive arousals and resultant ventilatory instability, we hypothesized that PLM-related arousals could be higher in patients with CompSAS than with OSA, and that perhaps PLM-related arousals could contribute to the high frequency of central apneas on CPAP. If this were the case, medical management of PLMS could potentially reduce the breathing instability of patients with CompSAS. To test this, we conducted a retrospective comparison of the PLM frequency (the PLM index, or PLMI) and PLM-related arousal frequency (the PLM arousal index, or PLMAI) in patients with complex sleep apnea.

\section{METHODS}

After gaining approval from the Mayo Clinic Institutional Review Board, we retrospectively reviewed the medical records of consecutive patients (age $\geq 19$ years) diagnosed with CompSAS at the Mayo Sleep Disorders Center from January of 2004 to June 2004 and compared them to a group of controls with OSA seen consecutively during January 2004. All patients and their sleep studies were evaluated by 
one of the team of board-certified sleep specialists. History, initial Epworth Sleepiness Scale (ESS) score, body mass index (BMI), presence of hypertension and other cardiovascular co-morbidities, polysomnographic data, and clinical diagnoses were recorded. We also gathered information from the medical record regarding therapies that may affect the prevalence of PLMS (dopaminergic agents, benzodiazepines, antidepressants, opioids, or anticonvulsants) and whether the sleep specialist diagnosed RLS.

Polysomnography was performed using a digital polygraph (NCI- LAMONT Medical Inc., Madison, Wisconsin or Bio-logic Systems Corp., Mundelein, Illinois). Sleep staging and arousals were scored according to standard methods [18, 19]. Surface electrodes were applied to the skin on both anterior tibialis muscles to detect muscle activity during sleep. PLMS were scored according to the criteria published by the former American Sleep Disorders Association [18]. Flow and respiratory effort was recorded using nasal pressure transducers plus uncalibrated respiratory impedance plethysmography (RIP) during the diagnostic phase. When CPAP was applied, the flow channel from the CPAP pneumotachometer plus RIP were used to analyze the air flow and respiratory effort.

Arousals were scored as respiratory-related arousals (RRA) if immediately preceded by any of the following: a cessation of airflow of at least 10 seconds' duration (apnea); a reduction of airflow of at least $30 \%$ and an accompanying drop in oxyhemoglobin saturation of at least $4 \%$ (hypopnea); any reduction of the amplitude of the nasal pressure transducer signal of at least 10 seconds' duration with an accompanying drop in saturation of 2-3\%; at least 3 consecutive snores of progressively increasing amplitude; or inspiratory flow limitation of at least 10 seconds' duration. The respira- tory-related arousal index (RRAI) was defined as the number of respiratory-related arousals per hour of sleep. Arousals were scored as periodic limb movement-related if they occurred concurrently with or within 2 seconds of the leg movement and there was no evidence for a simultaneous disordered breathing event. The periodic limb movement index (PLMI) was defined as the number of periodic limb movements per hour of sleep. The periodic limb movement arousal index (PLMAI) was defined as the number of arousals due to periodic limb movements per hour of sleep. The total arousal index (TAI) was defined as the total number of arousals per hour of sleep, encompassing those due to disordered breathing events, periodic limb movements, and indeterminate causes. CPAP titration was conducted as previously described, avoiding use of a pressure that was greater than needed to eliminate obstructive events [17].

Categorization of sleep disordered breathing was based upon the diagnostic portion of a split-night polysomnogram and the response to CPAP. Patients were diagnosed with OSA if the apnea-hypopnea index (AHI) was $\geq 5 \mathrm{hr}^{-1}$ and a level of CPAP was found that controlled breathing events. Patients were diagnosed with CompSAS if CPAP titration eliminated events defining OSA, but the residual central apnea index $\geq 5 \mathrm{hr}^{-1}$ or a Cheyne-Stokes breathing pattern (CSBP) became predominant and disruptive as previously described [14, 17, 20].

Results are expressed as medians (inter-quartile range) since most data were not normally distributed. Comparisons of continuous variables between groups were made with a 2sample t-test where appropriate and otherwise with the Mann-Whitney U test. Categorical variables were compared by Fisher's exact test (for $\mathrm{N}<5$ ) or the $\chi^{2}$ test $(\mathrm{N} \geq 5$ ).

Table 1. Demographics of Patients Included in the Study

\begin{tabular}{|c|c|c|c|}
\hline & CompSAS $(\mathbf{n}=\mathbf{8 8})$ & OSA $(n=112)$ & $\mathbf{p}$ \\
\hline Age & $60(47-70)$ & $59(51-69)$ & $0.55^{*}$ \\
\hline BMI & $31.7(27.6-36.9)$ & $32.4(28.4-38.2)$ & $0.30 *$ \\
\hline ESS & $11.0(7.0-16.0)$ & $10.0(7.0-15.0)$ & $0.32 *$ \\
\hline Presence of HTN & $50(57.5 \%)$ & $69(61.6 \%)$ & $0.47 * *$ \\
\hline Presence of A-fib & $14(16.1 \%)$ & $13(11.6 \%)$ & $0.40^{* *}$ \\
\hline $\operatorname{LVEF}(\%)$ & $60(55-65, \mathrm{n}=46)$ & $58(52-63, \mathrm{n}=53)$ & $0.19 *$ \\
\hline H/o RLS \# (\%) & $10(12.5)$ & $13(13.1)$ & $1^{* *}$ \\
\hline Use of benzodiazepines at time of study & $10(12.8)$ & $9(8.7)$ & 0.43 \\
\hline Use of SSRIs and tri-cyclic antidepressant drugs at time of study & $23(35.4)$ & $21(23.1)$ & 0.21 \\
\hline
\end{tabular}

CompSAS - complex sleep apnea syndrome, OSA - obstructive sleep apnea syndrome, BMI - body mass index, ESS - Epworth Sleepiness Scale, CHF - congestive heart failure, HTN - hypertension, A-fib- atrial fibrillation, LVEF - left ventricular ejection fraction from echocardiographic measurement, RLS - restless legs syndrome, SSRI- selective serotonin reuptake inhibitors. All data presented as medians (interquartile range).

*Mann-Whitney U test.

**Fisher's exact test. 


\section{RESULTS}

Our record review yielded 88 (71 males) patients with CompSAS with a median age of 60 years (interquartile range $47-70$ ), and 112 (85 males) patients with OSA with a median age of 59 years (interquartile range $51-69$ ). Demographic data are presented in Table 1. Both groups were similar with respect to ESS scores and presence of cardiovascular co-morbidities at initial presentation. RLS was diagnosed by the supervising sleep specialist in similar numbers of CompSAS and OSA patients. At the time of evaluation, significantly more patients with CompSAS (15/88, $17.0 \%)$ than patients with OSA $(3 / 112,2.7 \%)$ were taking opioid medications $(p<0.001$, Chi-square). Use of dopaminergic drugs, $\alpha$-blockers, $\beta$-blockers, benzodiazepines, and antidepressants (including selective serotonin reuptake inhibitors) was similar in both groups ( $p>0.05$ ) (Table 1).

Polysomnographic data are shown in Table 2. During the diagnostic portion, the TAI, RRAI, PLMI, and PLMAI were similar between CompSAS and OSA patients [40.9 vs. 39.8; 26.1 vs. $31.2 ; 4.3$ vs. 14.9 ; and 0 vs. 0.3 , respectively; all $\mathrm{p}>0.05]$. In contrast, during the CPAP titration, patients with CompSAS had a higher TAI [27.2 vs.16.6; $\mathrm{p}<0.001]$, RRAI [12.8 vs. $1.1 ; \mathrm{p}<0.001]$, but lower PLMI [0 vs. 12.6; $\mathrm{p}$ $=0.009]$ and PLMAI $[0$ vs. $0.8 ; \mathrm{p}=0.004]$ than patients with OSA. Distributions of the frequency of PLMI and PLMAI were skewed to the left, unimodal, and similar in both patient groups. The total sleep time, sleep efficiency, and the percent sleep in the supine position were similar in both groups.

\section{DISCUSSION}

The findings in our study are that PLMS are not common in patients with CompSAS and they occur with similar frequency to a matched population of patients with OSA. PLMrelated arousals are infrequent and thus are unlikely to play a significant role in destabilizing the breathing in CompSAS patients.

Between 3-15\% of patients with OSA develop a high frequency of central apnea and/or a disruptive CSBP after application of CPAP, a pattern called CompSAS [15-17, 21]. Much of our current knowledge on CompSAS comes from a few recently published retrospective studies $[13,14,17,20$, 22]. The underlying factors responsible for the development of central events on CPAP in patients with CompSAS are not well understood.

One could postulate that patients with CompSAS have at baseline reduced $\mathrm{CO}_{2}$ retention or enhanced ventilatory sensitivity to $\mathrm{CO}_{2}$ similar to that noted in some patients with idiopathic CSA, thus leading to respiratory instability after alleviation of upper airway obstruction [23]. Perhaps some feature of CPAP application enhances the respiratory responsiveness of the control system leading to destabilization of respiratory control. It is also possible that factors affecting respiratory loop gain or the respiratory mechanisms could play a role. One such possibility could be increased generalized anxiety or a more anxious response to medical equipment in these patients. Our data also show that nearly $20 \%$ of patients with CompSAS used opioids, agents known to be associated with central sleep apnea and altered respiratory control [24]. Opioid use could potentially be a risk factor for the development of CompSAS, though the mechanism is not understood; on the other hand, opioids are known to suppress PLMS. Our study design does not allow us to estimate the effect of opioid use on prevalence and characteristics of PLMS in our patients.

PLMS are repetitive, stereotypic movements of the limbs that are most common during the lightest stages of non-rapid eye movement sleep. PLMS may lead to autonomic arousals, cortical arousals, or awakenings. While PLMS may disrupt the sleep of bed partners, the clinical significance of the arousals caused by PLMS to the patient remains incompletely defined. On the one hand, Dickel et al. reported that PLMS are widespread in elderly but tend not to be manifested in sleep wake complaints [3]. Similarly, Chervin et al. observed that increased numbers of PLMS were not associated with objective sleepiness per multiple sleep latency testing or subjective sleepiness per the ESS [25].

Speculatively, the autonomic arousals caused by PLMS may be detrimental to co-morbid conditions. For example, there are reports of PLMS associated with congestive heart failure [8]. PLMS-induced arousals are thought to increase heart rate and left ventricular afterload via sympathetic activation, which may destabilize the failing heart $[10,11]$. Arousals from sleep may also induce hyperventilation, which acutely lowers pCO2 that may initiate and/or perpetuate Cheyne-Stokes breathing pattern [26, 27]. Using a similar line of reasoning, we hypothesized that an increased frequency of PLMS-induced arousals in CompSAS patients could theoretically lead to transient hyperventilation and explain their periodic breathing pattern on CPAP. Our data did not support this notion.

We had initially conceived this hypothesis based on our clinical impression that patients with CompSAS had an elevated PLMI. We considered several possible confounders that may have skewed the comparison data. The medications that are known to increase PLM frequency were not overrepresented in the OSA group, influencing results away from our hypothesis (Table 1). When patients with selective serotonin uptake inhibitors (SSRIs) were excluded, the differences in PLMI and PLMAI between OSA and CompSAS patients became statistically insignificant (Table 2). Although not directly studied, we do not believe that the PLMS distribute differently in REM vs NREM sleep in patients with CompSAS vs OSA. Thus, our clinical impression that PLM frequency might have been greater in patients with CompSAS appears to have been influenced by recall bias, as there was no significant difference in the PLMI between CompSAS and OSA patients. Nonetheless, the study results help to dispel the hypothesis that movement-related arousals are more common in and contribute to the development of CompSAS; only respiratory-related arousals are more common in patients with CompSAS. Alternative explanations for the apparent emergence of CSA on CPAP must be sought.

Our study has some potential limitations. All the patients in our study had undergone split-night polysomnography studies. This limits our ability to comment on sleep architectural details in these patients. PLMS unrelated to sleep disordered breathing may often occur during the first portion of sleep. Comparison of PLMS during the second portion of night in such case would not be optimal. However, since PLMS are distributed differently across portions of the night, this should not have unreasonably influenced our conclu- 
Table 2. Split-Night Polysomnography Results in Patients with Complex Sleep Apnea Syndrome and Obstructive Sleep Apnea Syndrome

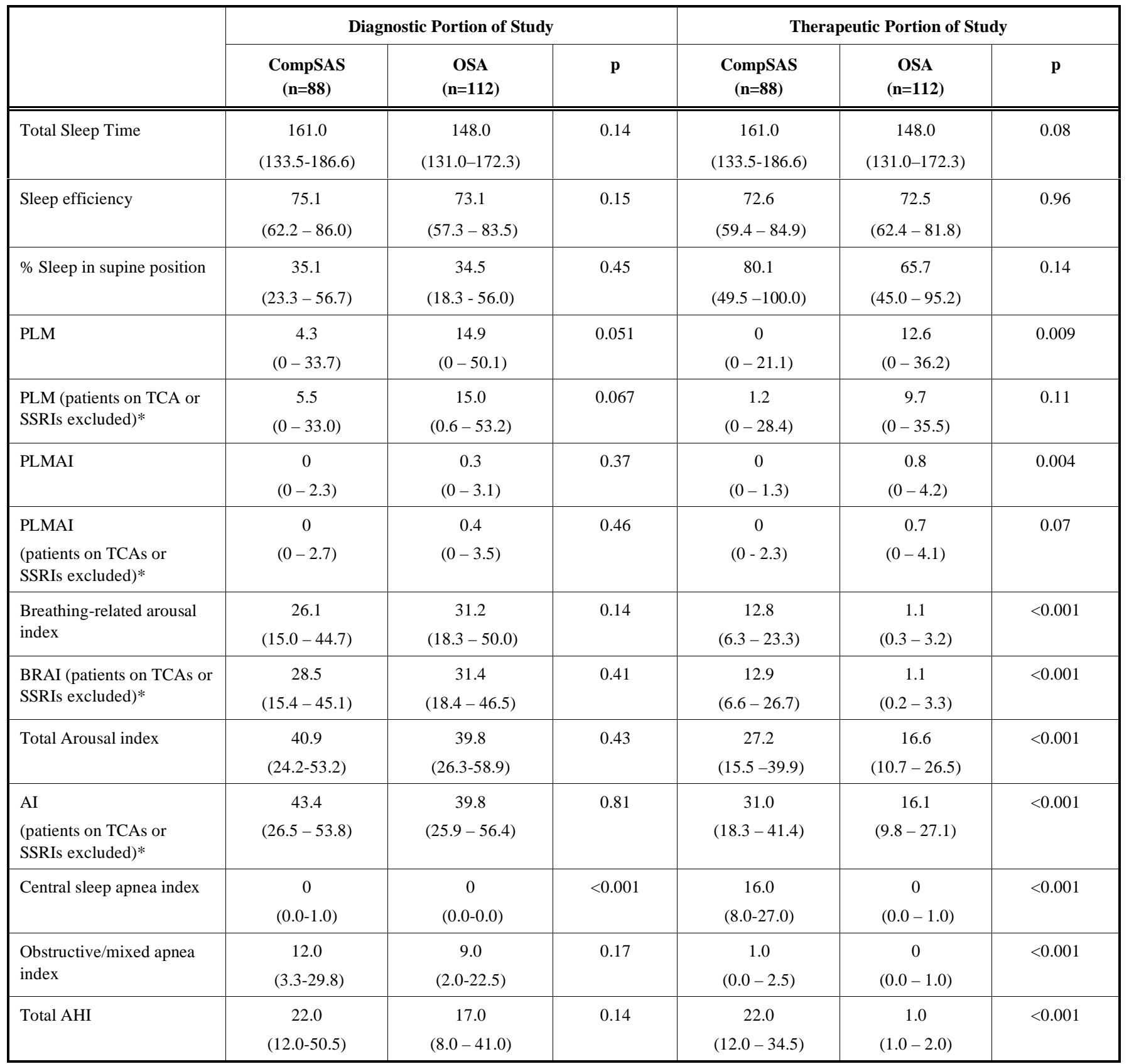

CompSAS - complex sleep apnea syndrome, OSA - obstructive sleep apnea syndrome, PLM - periodic limb movement index; PLMAI - periodic limb movement related arousal index, AI - arousal index, AHI - apnea-hypopnea index, SSRI - selective serotonin reuptake inhibitors, TCA - tricyclic antidepressants. All data presented as medians (interquartile range).

$* n=91$ for OSA and $n=65$ for CompSAS who were not taking TCAs or SSRIs.

sions since the diagnostic portion always came first and CPAP second, and the TST was similar in both groups (See Table 2). Finally, our study subjects were predominantly male, which limits generalization to female populations. Male gender is more prevalent in all reports of CompSAS, though gender as a risk factor has not been established.

\section{CONCLUSION}

PLMS are not more common in patients with CompSAS than in patients with OSA. PLM-related arousals do not seem to play a significant role in destabilizing respiratory control or in the pathogenesis of CompSAS. Replication of this finding in larger samples would strengthen this conclusion. Further research is warranted in order to gain a better understanding of the pathogenesis and treatment of this subtype of sleep disordered breathing.

\section{REFERENCES}

[1] Ancoli-Israel S, Kripke DF, Klauber MR, et al. Periodic limb movements in sleep in community-dwelling elderly. Sleep 1991; 14: $496-500$ 
[2] Bixler EO, Kales A, Vela-Bueno A, et al. Nocturnal myoclonus and nocturnal myoclonic activity in the normal population. Res Commun Chem Pathol Pharmacol 1982; 36: 129-40.

[3] Dickel MJ, Mosko SS. Morbidity cut-offs for sleep apnea and periodic leg movements in predicting subjective complaints in seniors. Sleep 1990; 13: 155-66.

[4] Ancoli-Israel S, Kripke DF, Mason W, et al. Sleep apnea and periodic movements in an aging sample. J Gerontol 1985; 40: 419-25.

[5] Montplaisir J, Boucher S, Poirier G, et al. Clinical, polysomnographic, and genetic characteristics of restless legs syndrome: a study of 133 patients diagnosed with new standard criteria. Mov Disord 1997; 12: 61-5.

[6] Lapierre O, Montplaisir J. Polysomnographic features of REM sleep behavior disorder: development of a scoring method. Neurology 1992; 42: 1371-74.

[7] Wittig R, Zorick F, Piccione P, et al. Narcolepsy and disturbed nocturnal sleep. Clin Electroencephalogr 1983; 14: 130-4.

[8] Hanly PJ, Zuberi-Khokhar N. Periodic limb movements during sleep in patients with congestive heart failure. Chest 1996; 109: 1497-502.

[9] Javaheri S. Sleep disorders in systolic heart failure: a prospective study of 100 male patients. The final report. Int J Cardiol 2006; 106: 21-8.

[10] Ali NJ, Davies RJ, Fleetham JA, et al. Periodic movements of the legs during sleep associated with rises in systemic blood pressure. Sleep 1991; 14: 163-5.

[11] Sforza E, Nicolas A, Lavigne G, et al. EEG and cardiac activation during periodic leg movements in sleep: support for a hierarchy of arousal responses. Neurology 1999; 52: 786-91.

[12] Stoohs RA, Blum HC, Suh BY, et al. Misinterpretation of sleepbreathing disorder by periodic limb movement disorder. Sleep Breathing 2001; 5: 131-7.

[13] Gilmartin GS, Daly RW, Thomas RJ. Recognition and management of complex sleep-disordered breathing. Curr Opin Pulm Med 2005; 11: 485-93.

[14] Thomas RJ, Terzano MG, Parrino L, et al. Obstructive sleepdisordered breathing with a dominant cyclic alternating pattern--a recognizable polysomnographic variant with practical clinical implications. Sleep 2004; 27: 229-34.

[15] Lehman S, Antic NA, Thompson C, Catcheside PG, Mercer J, McEvoy RD. Central sleep apnea on commencement of continuous positive airway pressure in patients with a primary diagnosis of obstructive sleep apnea-hypopnea. J Clin Sleep Med 2007; 3(5): 4626.

[16] Ando SI, Ishitobi Y, Yagi T, et al. Prevalence of complex sleep apnea syndrome in Japan. Sleep Biol Rhythms 2008; 6: 190-2.

[17] Morgenthaler TI, Kagramanov V, Hanak V, et al. Complex sleep apnea syndrome: is it a unique clinical syndrome? Sleep 2006; 29: 1203-9.

[18] Recording and scoring leg movements. The atlas task force. sleep 1993; 16: 748-59.

[19] EEG arousals: scoring rules and examples: a preliminary report from the Sleep Disorders Atlas Task Force of the American Sleep Disorders Association. Sleep 1992; 15: 173-84.

[20] Thomas RJ, Daly RW, Weiss JW. Low-concentration carbon dioxide is an effective adjunct to positive airway pressure in the treatment of refractory mixed central and obstructive sleep-disordered breathing. Sleep 2005; 28: 69-77.

[21] Sleep-related breathing disorders in adults: recommendations for syndrome definition and measurement techniques in clinical research. The Report of an American Academy of Sleep Medicine Task Force. Sleep 1999; 22: 667-89.

[22] Pusalavidyasagar SS, Olson EJ, Gay PC, et al. Treatment of complex sleep apnea syndrome: a retrospective comparative review. Sleep Med 2006; $7:$ 474-9.

[23] Solin P, Roebuck T, Johns DP, et al. Peripheral and central ventilatory responses in central sleep apnea with and without congestive heart failure. Am J Respir Crit Care Med 2000; 162: 2194-200.

[24] Wang D, Teichtahl H. Opioids, sleep architecture and sleepdisordered breathing. Sleep Med Rev 2007; 11: 35-46.

[25] Chervin RD. Periodic leg movements and sleepiness in patients evaluated for sleep-disordered breathing. Am J Respir Crit Care Med 2001; 164: 1454-58.

[26] Somers VK, Dyken ME, Clary MP, et al. Sympathetic neural mechanisms in obstructive sleep apnea. J Clin Invest 1995; 96: 1897-904.

[27] Horner RL, Rivera MP, Kozar LF, et al. The ventilatory response to arousal from sleep is not fully explained by differences in $\mathrm{CO}(2)$ levels between sleep and wakefulness. J Physiol 2001; 534: 881-90.

Received: January 05, 2009

Revised: January 28, 2009

Accepted: April 01, 2009

(C) Pusalavidyasagar et al.; Licensee Bentham Open.

This is an open access article licensed under the terms of the Creative Commons Attribution Non-Commercial License (http://creativecommons.org/licenses/by-nc/3.0/) which permits unrestricted, non-commercial use, distribution and reproduction in any medium, provided the work is properly cited. 